\title{
Why are CMEs large-scale coronal events: nature or nurture?
}

\author{
L. van Driel-Gesztelyi ${ }^{1,2,3}$, G. D. R. Attrill ${ }^{1}$, P. Démoulin ${ }^{2}$, C. H. Mandrini ${ }^{4}$, and L. K. Harra ${ }^{1}$ \\ ${ }^{1}$ University College London, Mullard Space Science Laboratory, Holmbury St. Mary, Dorking, Surrey, RH5 6NT, UK \\ ${ }^{2}$ Observatoire de Paris, LESIA, FRE 2461(CNRS), 92195 Meudon Principal Cedex, France \\ ${ }^{3}$ Konkoly Observatory of Hungarian Academy of Sciences, Budapest, Hungary \\ ${ }^{4}$ Instituto de Astronomía y Física del Espacio, CONICET-UBA, CC. 67, Suc. 28, 1428 Buenos Aires, Argentina
}

Received: 4 October 2007 - Revised: 17 January 2008 - Accepted: 18 February 2008 - Published: 15 October 2008

\begin{abstract}
The apparent contradiction between small-scale source regions of, and large-scale coronal response to, coronal mass ejections (CMEs) has been a long-standing puzzle. For some, CMEs are considered to be inherently large-scale events - eruptions in which a number of flux systems participate in an unspecified manner, while others consider magnetic reconnection in special global topologies to be responsible for the large-scale response of the lower corona to CME events. Some of these ideas may indeed be correct in specific cases. However, what is the key element which makes CMEs large-scale? Observations show that the extent of the coronal disturbance matches the angular width of the CME - an important clue, which does not feature strongly in any of the above suggestions. We review observational evidence for the large-scale nature of CME source regions and find them lacking. Then we compare different ideas regarding how CMEs evolve to become large-scale. The large-scale magnetic topology plays an important role in this process. There is amounting evidence, however, that the key process is magnetic reconnection between the CME and other magnetic structures. We outline a CME evolution model, which is able to account for all the key observational signatures of large-scale CMEs and presents a clear picture how large portions of the Sun become constituents of the CME. In this model reconnection is driven by the expansion of the CME core resulting from an over-pressure relative to the pressure in the CME's surroundings. This implies that the extent of the lower coronal signatures match the final angular width of the CME.
\end{abstract}

Keywords. Solar physics, astrophysics and astronomy (Flares and mass ejections)

Correspondence to: L. van Driel-Gesztelyi

(lvdg@mssl.ucl.ac.uk)

\section{Introduction}

The apparent contradiction between small-scale source regions of, and large-scale coronal response to, coronal mass ejections (CMEs; cf. Fig. 1) has been highlighted by Klimchuk (2001): "Coronagraph observations suggest that the horizontal scale of the opened field can be many times greater than that of the reconnection arcade and this may be difficult to reconcile with the geometry of the [existing] model[s]."

High-quality data (principally SOHO) coupled with some new ideas have brought us closer to solving this puzzle. In this work we outline and compare different (and controversial) approaches. One side suggest that CMEs are inherently large-scale events, so that their energy supply, initiation and final angular width all originate from a large-scale region, i.e. being large-scale is their nature (e.g. Zhang et al., 2007; Wang et al., 2007; Zhukov and Veselovsky, 2007). The other side consider that CMEs start small-scale and then evolve to become large-scale events. Thus CMEs may become large-scale even in the low corona due to interaction between the expanding magnetic structure of the CME and other low-coronal magnetic structures (e.g. Maia et al., 1999; Pohjolainen et al., 2001; Bemporad et al., 2005; Attrill et al., 2007a; Mandrini et al., 2007; Moore and Sterling, 2007). In this case, CMEs become large-scale by nurture - they are not large-scale by nature.

The paper is organised as follows: in Sect. 2 we make an overview of large-scale low-coronal signatures of CME, whose extent we link to the angular width of the CME. In Sect. 3 we discuss arguments for the inherently large-scale nature of CME source regions. In Sect. 4 we show some examples how CMEs can evolve to become large-scale in the low corona. In Sect. 5 we discuss what determines the angular width of CMEs and outline the consequences of magnetic interaction between the expanding CME and surrounding magnetic structures. We present our conclusions in Sect. 6.

Published by Copernicus Publications on behalf of the European Geosciences Union. 


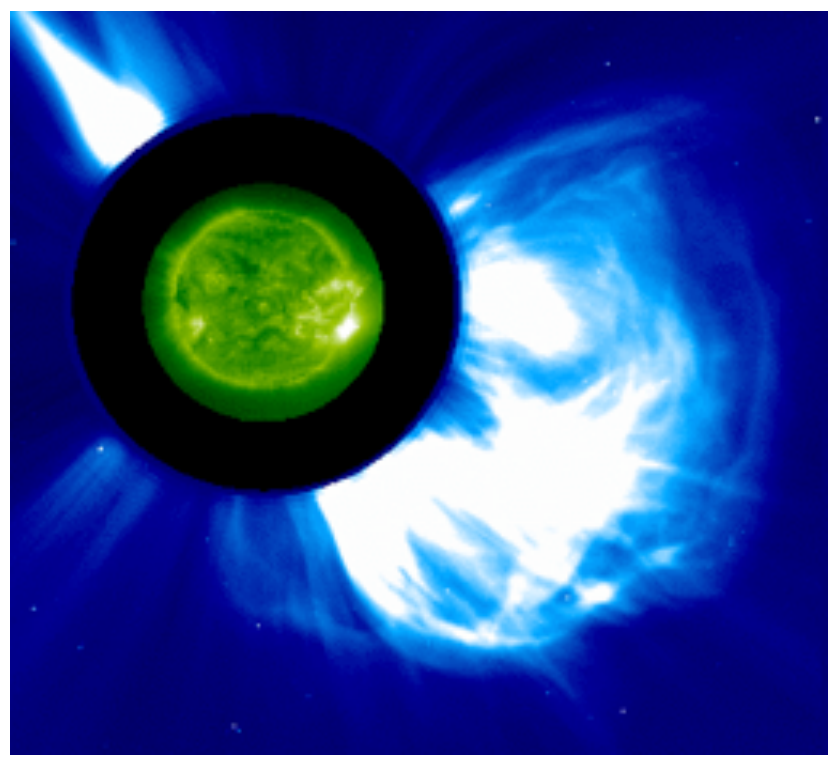

Fig. 1. CME on the 4th November 2003 (EIT: 20:00 UT, LASCO/C2: 20:06 UT). Illustrating the contradiction between small-scale source region (the bright flaring region on the West limb) and the large extent of the CME.

\section{Large-scale low coronal signatures of CMEs}

2.1 Summary of the large-scale low coronal CME signatures

Besides activity seen in the core source region of CMEs e.g. filament eruption, flare loop arcade, and X-ray/EUV double dimming (for a review see Hudson and Cliver, 2001) there are many different large-scale low coronal signatures (mainly seen in EUV and soft X-rays) associated with CMEs, including:

(i) Wide-spread coronal dimming (Hansen et al., 1974; Hudson et al., 1996; Thompson et al., 2000a; Attrill et al., 2007a,b; Zhukov and Veselovsky, 2007; Mandrini et al., 2007).

(ii) Wide-spread activity of non-thermal radio emission (Tang and Moore, 1982; Pick et al., 1998; Maia et al., 1999; Pohjolainen et al., 2001, 2005; Pick et al., 2006; Wen et al., 2006)

(iii) Disappearance of large-scale loops (including TransEquatorial Loops (TELs); (Delannée and Aulanier, 1999; Khan and Hudson, 2000; Pohjolainen et al., 2001)).

(iv) Coronal (EIT) wave (Moses et al., 1997; Thompson et al., 1998).

(v) Stationary brightenings distant to the flaring region (Delannée, 2000; Delannée et al., 2007).

(vi) Disturbances along coronal hole boundaries (Hudson et al., 1996; Attrill et al., 2006; Veronig et al., 2006; Harra et al., 2007a).

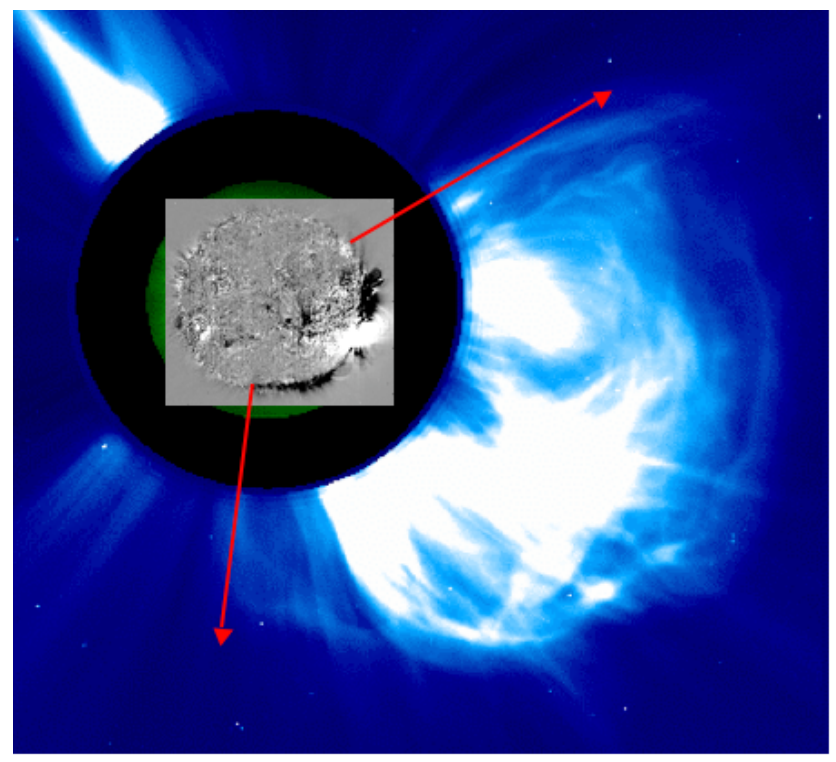

Fig. 2. CME on the 4 November 2003 (EIT and LASCO/C2: same times as in Fig. 1). Showing how the spatial extent of dimming in the low corona approximately matches the angular width of the CME. The SOHO/EIT image is a base difference image, i.e. a preevent image was subtracted from it.

The above signatures do not all necessarily appear in one given event and they mainly accompany CMEs with large final angular width. There is one important clue, which envelops all the above signatures, first noticed by Webb et al. (1997) and Thompson et al. (2000a), namely that the total extent of the lower-coronal disturbances (i-vi) match the angular width of the CME (which is best seen in CMEs erupting from close to the solar limb; see e.g. Fig. 2). This observation should be kept in mind when one considers possible solutions to the problem.

\subsection{Wide-spread coronal dimming}

We rely on on-disc observations of the corona to give information about the formative stages of a CME since coronagraph observations are restricted to off-limb only. The manifestation of dimmings (depletions in intensity of coronal plasma) are widely acknowledged to occur in the corona in association with CMEs (Hansen et al., 1974; Hudson et al., 1995, 1997). Dimmings can, a priori, be due to temperature effects (Thompson et al., 1998; Chertok and Grechnev, 2003). However, since the dimmings appear simultaneously at the same spatial location, in a broad range of wavelengths (EUV, soft X-rays - see e.g. Zarro et al., 1999) and temperatures, they primarily appear to be due to density depletion rather than being a temperature effect. Indeed this interpretation is consistent with an evacuation of plasma (Hudson et al., 1996) upon eruption of the local magnetic field. Work 


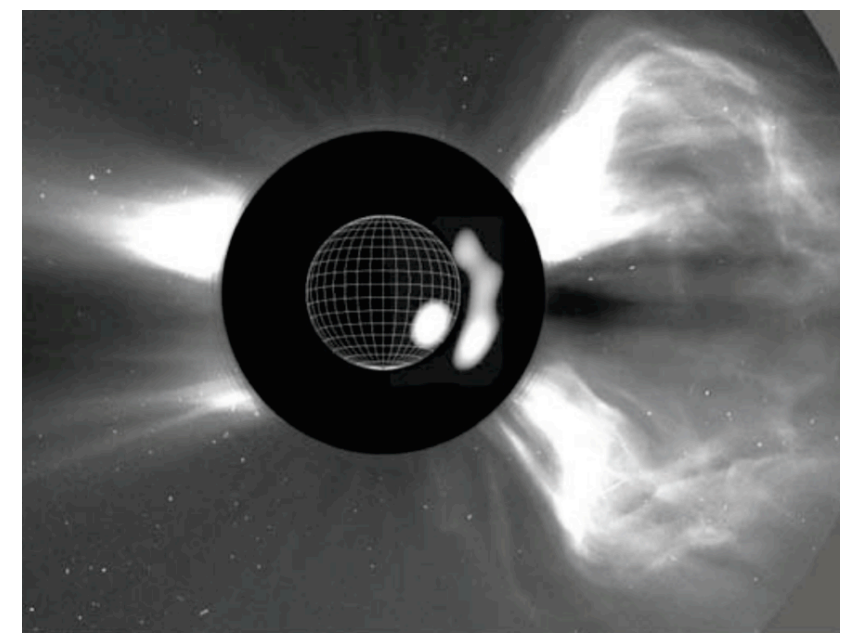

Fig. 3. From Maia et al. (1999) on 6 November 1997 CME event showing that the angular width of the CME is comparable to the spatial extent of the radio emission (i.e. the bright regions on the solar disc and over the west limb).

by Harra and Sterling (2001) directly supports this interpretation. Their analysis of SOHO/CDS (Coronal Diagnostic Spectrometer) data showed blue-shifted mass motion in coronal dimming regions, consistent with the interpretation that dimming is indeed due to plasma evacuation. Recently, Hinode/EIS has confirmed this outflow from dimming regions (Harra et al., 2007b). Harrison and Lyons (2000) found that such dimming is mainly caused by a loss of plasma at a temperature $\approx 10^{6} \mathrm{~K}$.

\subsection{An example of radio observations on the limb}

Radio observations are very sensitive indicators of sites of non-thermal electron acceleration in the solar corona, therefore they can show the extent of lower coronal disturbance in a CME. The extent of radio continuum emission has been observed to match the angular extent of the departing CME on 6 November 1997 (Fig. 3, Maia et al., 1999). The authors note that the sites of radio emission are located in regions of interaction between the CME source region and adjacent loop structures, corresponding to electron acceleration sites distinct from the flaring region.

2.4 An example of radio observations on the disc - linking dimming and radio emission

For CMEs which are initiated close to the disc centre, it is difficult to estimate the true angular width of the CME, however, details of the large-scale lower coronal signatures can be investigated. An example is shown in Fig. 4. The $236 \mathrm{MHz}$ radio emission appears just before the dimming is observed in EIT data (Pohjolainen et al., 2001). For dimming to occur, as discussed in Sect. 2.2, plasma needs to be evac-

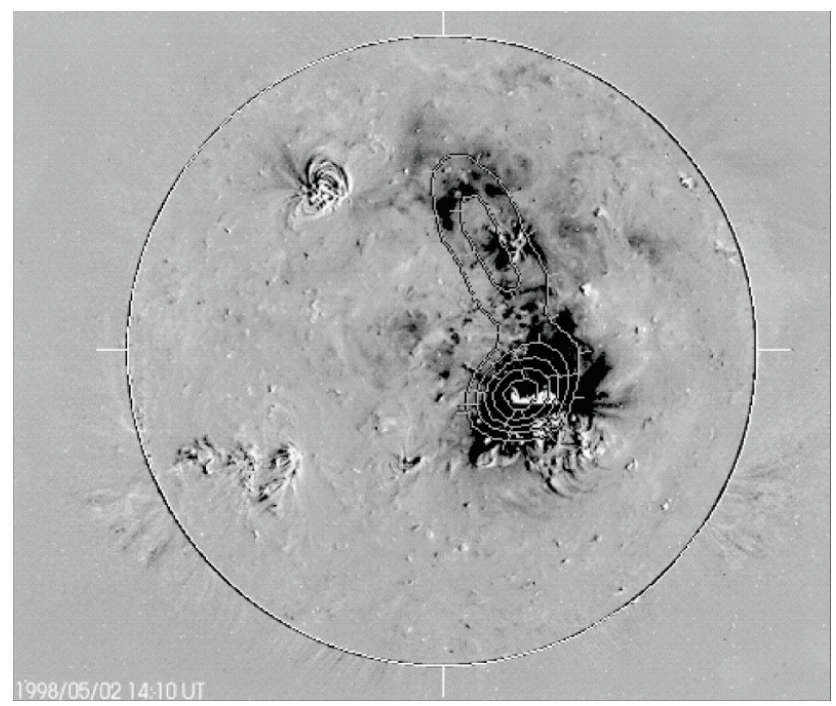

Fig. 4. From Pohjolainen et al. (2001) on 2 May 1998 CME event. EIT difference image showing coronal dimming (black regions) overlaid with $236 \mathrm{MHz}$ Nancay Radio Telescope (NRT) moving continuum radio source isocontours. Note how well the nonthermal radio emission matches the location where the dimmings subsequently appear (EIT difference image: 14:10-13:41 UT; NRT 236 MHz contours: 13:48:21 UT).

uated, most plausibly by the magnetic field being "opened" (i.e. being extended upward to scales much larger than the gravitational scale height of the corona $\approx 100 \mathrm{Mm}$ ). Is the $236 \mathrm{MHz}$ emission a signature of the beginning of this "opening" process?

How exactly does the field "open up", i.e. how does it become part of the CME over such a large area as evidenced by the dimming footprint and non-thermal radio emission? We discuss this question further in the following two sections.

\section{Are CME source regions large-scale?}

One of the possible solutions to the contradiction between small-scale source-region and large-scale CME is to consider that the source region of a CME extends well beyond the initiation site, frequently marked by a (two-ribbon) flare. This view would make CMEs large-scale by nature.

This view is endorsed by Wang et al. (2007) and Zhang et al. (2007), who write: "CME source regions must include the large-scale extent of a CME (spanning up to $100-140^{\circ}$ on the disc), not just the initiation site." and "A single active region with its flare activity may not be capable of producing a CME with large angular width."

Zhukov and Veselovsky (2007) also argue for the nonlocal nature of the CME mechanism when stating: "We can only speculate about subphotospheric drivers and the accumulation of free energy in the solar atmosphere that may be 

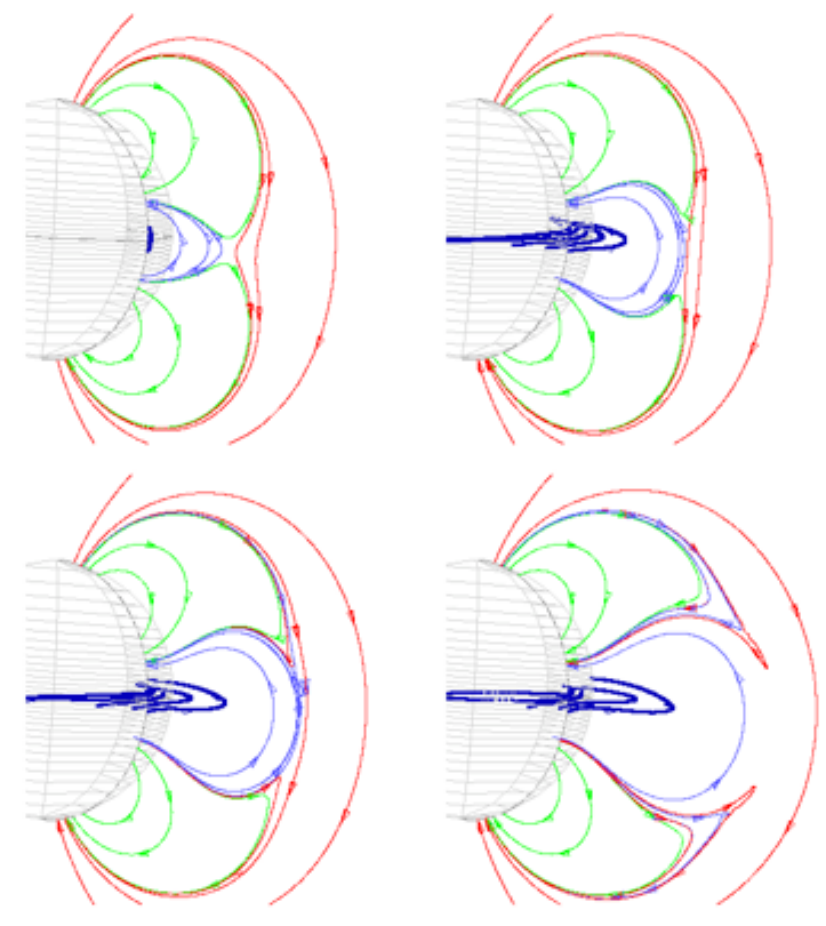

Fig. 5. The breakout model involves the large-scale magnetic environment in CME initiation, but neither the CME width is defined by the large-scale field nor it leads to wide-spread dimming (Antiochos et al., 1999).

involved in the global CME initiation and development. They may be related to global electric currents and circuits connecting solar interior and corona."

Are CMEs inherently large-scale events - eruptions in which a number of flux systems participate in an unspecified manner or are they linked through the solar interior?

Zhou et al. (2007) argue that quasi-simultaneous flux emergence in the large complex of activity related to the CME/flare-prolific period of October-November 2003 supports the idea of sub-photospheric link. They find the same sign of helicity in the three major ARs of the complex and a filament channel which thread through them, linking them all. They suggest: "The instability of the large-scale flux system, and/or its interaction with other magnetic systems, may be the origin of the prolific CME initiation."

Zhang et al. (2007) invoke the breakout model (Antiochos et al., 1999) as evidence for the non-local nature of CME initiation. In the breakout model the large-scale quadrupolar topology is essential for the CME initiation mechanism. However, the erupting field is still bipolar and localized in the core source region. Thus the breakout mechanism does not produce the characteristic large-scale coronal signatures of a CME (see Sect. 2.1) nor can it be invoked as an evidence for the intrinsically large-scale nature of CME source regions. This is because the external field lines in the quadrupolar configuration (after reconnection with the expanding core field) will close down forming two lobes on both sides of the core field (Fig. 5). As a result of the reconnection, these external field lines form shorter loops and therefore they may brighten, but little or no dimming appears: the plasma trapped in these shorter loops will become more, and not less, dense - see e.g. the careful study of a slowly erupting quietsun filament by Sterling and Moore (2004).

It is noteworthy that most of the observational evidence of the breakout model was found in so-called "lateral breakout" configurations within complex ARs (Aulanier et al., 2000; Gary and Moore, 2004; Harra et al., 2005; Williams et al., 2005), with no significant involvement of large-scale external magnetic fields (though for an exception see Sterling and Moore, 2004). On the other hand, in the lateral break-out the large-scale upper arcade could be de-stabilised and therefore can plausibly lead to an increase of the spatial extent of instability. Therefore we rather consider it as a model supporting the view that CMEs become large-scale and will discuss it in more detail in Sect. 4.4 .

\section{How can CMEs become large-scale in the low corona?}

Since CMEs are magnetic structures and the solar magnetic field is ubiquitous, there is a good chance that the expanding CME structure will meet overlying loops forcing them to expand ("opening" them). Magnetic reconnection may also be part of the interaction process, since it is probable that the field lines of the CME and those of the overlying loops are non-parallel (the extreme case being when the magnetic fields are anti-parallel).

\subsection{Interaction with overlying and trans-equatorial loops}

The "giant arches" discovered by Švestka et al. (1982) in SMM data and analysed using Yohkoh/SXT observations (Švestka et al., 1995) included some cases of erupting transequatorial loop (TEL), and provided possible evidence for large-scale coronal restructuring associated with CMEs.

Are large-scale connections, such as TELs, involved in a CME provide evidence for the large-scale nature of CMEs? Or are they simply get involved through an inevitable interaction of the CME with its surroundings?

The first and still one of the best-documented CME-TEL interactions was described by Manoharan et al. (1996). On 25 October 1994 an expanding soft X-ray (Yohkoh/SXT data) sigmoid was seen close to the equator in the Southern Hemisphere, associated with a long-duration flare event (LDE). The expansion was accompanied by a series of radio bursts (NRT data), which closely followed the loop expansion both in time and space. Simultaneously with the nonthermal radio bursts, the appearance of two remote X-ray brightenings was seen in opposite hemispheres, followed by the formation of two coronal holes above those weak (quiet) 


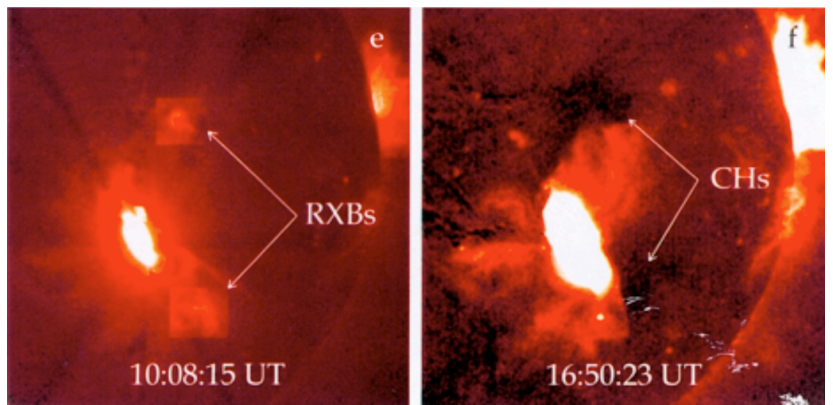

Fig. 6. From Manoharan et al. (1996) showing the consequences of interaction between a CME and overlying TELs on 25 October 1994: formation of X-ray brightenings (RXBs, left panel) followed by the appearance of coronal dimmings ( $\mathrm{CHs}$ ) at the same location. Extensive loop formation between the flaring AR and the edge of the dimming regions (Yohkoh SXT data) was also observed.

magnetic regions of opposite polarity (Fig. 6). During the six hour long gradual phase of the flare, new X-ray loop connections developed among the AR and the remote quiet regions. These series of events were interpreted as signatures of a large-scale reconnection process between the erupting core field and overlying trans-equatorial loops connecting quietSun regions. However, not all the erupting field reconnected. The most external part of the overlying large-scale fields was pushed out (forced to expand) into the solar wind by the expanding twisted loops, leading to the formation of the coronal holes.

In other cases TELs, rooted close to the initiation site of the CME (as opposed to lying over the initiation site), were observed to disappear. Khan and Hudson (2000) described a series of eruption and re-formation of SXR trans-equatorial loops associated with the CME-prolific NOAA AR 8210 during May 1998. At least four such events happened: on 2, 6, 8, and 9 May. They linked the eruption of the trans-equatorial loops to the occurrence of CMEs having larger angular scales than that of their associated flares. In these events the erupting loop systems became part of the associated CMEs. These events were also associated with major flares and with global travelling emission visible in soft X-rays and type II radio bursts. Similar conclusions were reached by Pohjolainen et al. (2001, 2005, cf. Fig. 4).

Glover et al. (2003) investigated a sample of 18 TELs and found that 10 (thus only about half) were associated with flaring and CME onset originating from the AR to which they were connected. They found a variety of different signatures, including the formation of a bright hot (SXR) cusp interconnecting ARs with dimming gradually spreading along some TELs (cf. Fig. 7). This observation provides support for the scenario that CMEs are locally initiated, and during their development they expand to involve other large-scale magnetic structures.

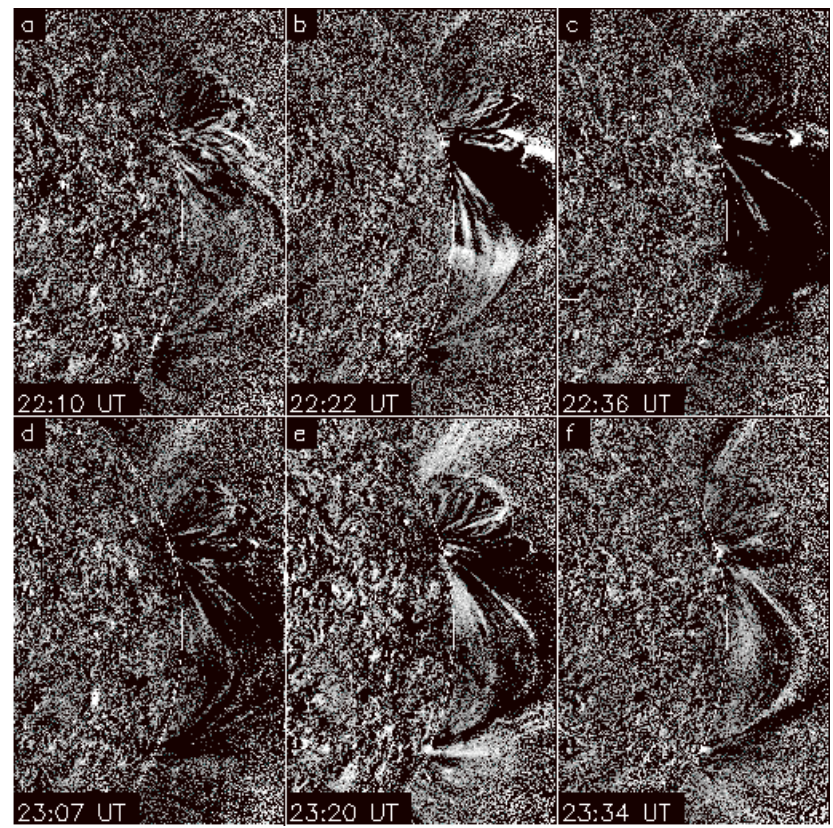

Fig. 7. From Glover et al. (2003) showing how low-coronal dimming evolves and spreads due to the involvement of a transequatorial loop system (TEL) in the CME process on 29 May 1998. EIT $195 \AA$ difference images show that a dimming appears first around the flaring AR in the north, then gradually spreads along the TEL while its distant footpoint brightens and an SXR cusp appears above the TEL, suggestive of the post-"opening" reconnection process. Flaring behaviour of a TEL has also been found by Harra et al. (2005).

The cause of TEL eruption was clear in the case shown by Manoharan et al. (1996) since there it was initiated by CME eruption from under the TEL. However, how is the TEL eruption initiated in the case where the TEL is rooted in the vicinity of the AR where a CME is initiated?

Delannée and Aulanier (1999) analyse the large-scale magnetic topology on 3 November 1997 around the CMEprolific AR 8100 which was connected by TELs to AR 8102 . They find a so-called bald-patch magnetic topology in the vicinity of the initiation site AR81000 (i.e. field aligned tangent to the photosphere). They observe the fast "opening" of the TELs, which was evidenced by coronal dimming around their foot-points. They propose that this "opening" was driven by low-lying sheared field lines overlying the baldpatch as TELs were liberated from line-tying by the baldpatch reconnection.

An interesting aspect of CME-TEL relationship was described by Harra et al. (2007a) and Wang et al. (2007): CMEs can actually form TELs, not only disrupt them. In the period 3-7 November 2004 several CMEs were initiated in the complex AR10696, which through magnetic reconnection with "open" field lines of a coronal hole (CH) situated in the opposite hemisphere, formed and re-formed an impressive system of TELs. 


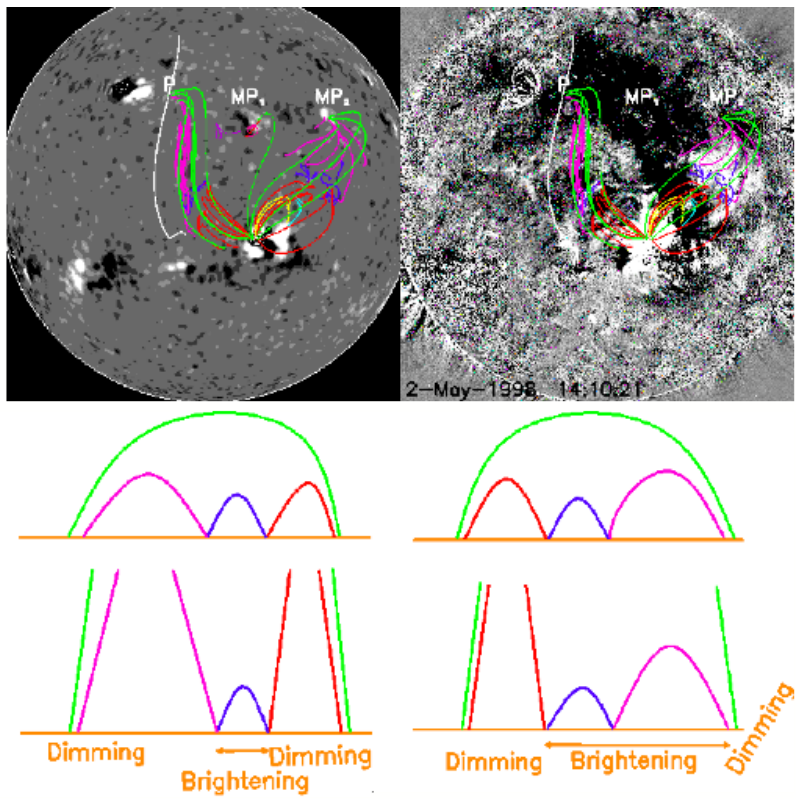

Fig. 8. From Delannée et al. (2007) linking dimmings and distant stationary brightenings to location of QSLs in the initial magnetic large-scale topology. The CME is initiated at the southern end of these loops. The cartoons in the lower panel, which have the same colour code as the field lines in the upper panel, illustrate how the stationary brightenings are linked to the expansion of the TEL field lines. The cause of expansion of the TELs, however, remains unclear.

In summary, the eruption of large-scale loops can indeed contribute significantly to making CMEs large-scale in the low corona: they lead to remote brightenings, non-thermal radio bursts and coronal dimming extending well beyond the vicinity of the AR. However, the eruption of TELs alone cannot explain the so-called "global CMEs" described e.g. by Zhukov and Veselovsky (2007) and their associated widespread diffuse dimmings (cf. Fig. 1).

\subsection{Importance of the large-scale magnetic topology}

The introduction of the analysis of the large-scale magnetic topology was an important step in understanding how CMEs become large-scale in the lower corona. In a pioneering study Delannée and Aulanier (1999) linked the formation of largescale dimming to reconnection (in a bald-patch topology, as already described in Sect. 4.1). More recently, Delannée et al. (2007) linked dimmings and distant stationary brightenings to the locations of separatrices and quasi-separatrix layers (QSLs) in the large-scale topology (see Fig. 8). They also linked EIT and Moreton waves to Joule heating resulting from the generation of electric currents in the vicinity of separatrices and QSLs, while the magnetic field lines are "opening" during a CME. In another recent work by Zhang et al. (2007), the large-scale topology was investigated in four large-scale CME events (14 July 2000, 28 October 2003, 7 November 2004, and 15 January 2005). They found that each CME event involved interaction of more than ten sets of magnetic-loop systems, covering a wide range of solar longitudes and latitudes. A saddle-field configuration (i.e. the magnetic configuration in the vicinity of a null point) was found to be present for all these four major CME events. The authors conclude that interaction of multiple flux-loop systems is required for a major $\mathrm{CME}$.

An interesting work by Wen et al. (2006) links large-scale magnetic topology to observation of radio bursts. They show that non-thermal radio bursts (understood to be signatures of reconnection in the region of separatrices) appear in phase with, and at the same location as, the dimming regions and spread over the solar disc with velocity $\approx 300-400 \mathrm{~km} \mathrm{~s}^{-1}$ in $17 \pm 2$ min (though individual bursts shift with $v \approx 700$ $1000 \mathrm{~km} \mathrm{~s}^{-1}$ ). The authors propose that these "topology waves" represent successive topology changes from closed to "open" field configurations.

However, it is not clear in all these papers how exactly the QSLs and multiple loop systems contribute to the large-scale expansion involved in these large-scale CMEs.

\subsection{Role of magnetic reconnection}

Monique Pick and co-authors starting as early as 1998, introduced the idea that CMEs become large-scale in the low corona due to magnetic reconnection between adjacent magnetic systems. Based on multi-wavelength, prominently metric radio, data of the 9 July 1996 CME event Pick et al. (1998) concluded: "The analysis of the time sequence of the radio continuum reveals the existence of distinct phases associated with distinct reconnection processes and magnetic restructuring of the corona." The excellent spatial association found between the position and extension of the radio sources and the angular extent of the associated (limb) CME seen by LASCO (Fig. 3) was an important further step (Maia et al., 1999). Another clue came from the analysis of on-disc CMEs, linking non-thermal radio burst locations to the subsequent development of dimming regions (Fig. 4, Pohjolainen et al., 2001). Since then, the explanation of the observed wide-spread radio activity due to loop-loop interactions, resulting in coronal re-structuring in a multipolar magnetic field, and ultimately formation of a largescale CME, featured in many works by the Meudon group (see references within Pick et al., 2006).

There are many CMEs which, in the outer corona, are laterally far offset from the initiation (flare) site (Harrison, 1986). Moore and Sterling (2007), revisited the streamerpuff CME analysed by Bemporad et al. (2005), showing how CMEs can be produced by the blowout of a large quasipotential arch by a compact eruption occurring at one of the legs of the arch through magnetic reconnections between adjacent magnetic arcades (Fig. 9). The authors apply this reconnection scenario to several cases of published 
trans-equatorial CMEs produced in tandem with an ejective flare or filament eruption that was far offset from directly under the CME. This work shows how a large-scale CME can originate from a small source region and how it becomes large-scale through reconnection with a neighbouring active region. They emphasise the role of overlying streamer magnetic fields and neighbouring arcades.

\subsection{Breakout - possibility for chain-reconnection}

A very interesting double CME event on 15 July 2004 was analysed by Gary and Moore (2004) and Harra et al. (2005), which was interpreted to be due to both central and lateral break-out occurring successively. In the case of a lateral break-out, the sheared core field is located in one of the side lobes (as opposed to being in the centre as in Fig. 5). AR 10030 had a quadrupolar magnetic configuration, which contained two filament channels (i.e. highly sheared core fields): one in the center and another in one of the side-lobes. First, a quadrupolar reconnection (manifested by a confined quadrupolar flare) removed confining magnetic field lines above the central filament, which erupted. The relaxation reconnection, in turn, weakened field lines stabilising the highly sheared filament in the side lobe, which then erupted, producing a second CME.

The above scenario illustrates the potential of the breakout mechanism in leading to "sympathetic" eruptions.

\subsection{Reconnection with coronal holes}

Coronal holes are another type of magnetic structure that, upon interaction and reconnection with a CME can dramatically alter the global topology of the CME. Since coronal holes are very frequently present in the vicinity of CME initiation sites (85-95\%, see Hewish and Bravo, 1986; Bravo, 1995; Bravo et al., 1999), this type of interaction should be a frequent signature of large-scale CMEs. A striking case (13 November 1994 CME) was presented by Hudson et al. (1996), who found CME-related disturbances near the boundaries of both(!) polar coronal holes. In the 17 January 2005 CME Veronig et al. (2006) found that interaction between CME and a coronal hole depended on the orientation of the polar $\mathrm{CH}$ boundary relative to the direction of CME expansion.

Other well-observed examples include the 12 May 1997 (Attrill et al., 2006; Crooker and Webb, 2006) and the 67 November 2004 CME events (Harra et al., 2007a), when long-lasting magnetic reconnection was observed between the expanding CME and magnetic fields of a neighbouring coronal hole. Another more recent example, using STEREO/EUVI data highlights interaction with a low latitude disk coronal hole. In this example, reconnection in the upper corona between the expanding CME and the "open" coronal hole magnetic field hinders the lateral expansion of a limb CME across the solar disk (Attrill et al., 2007b).
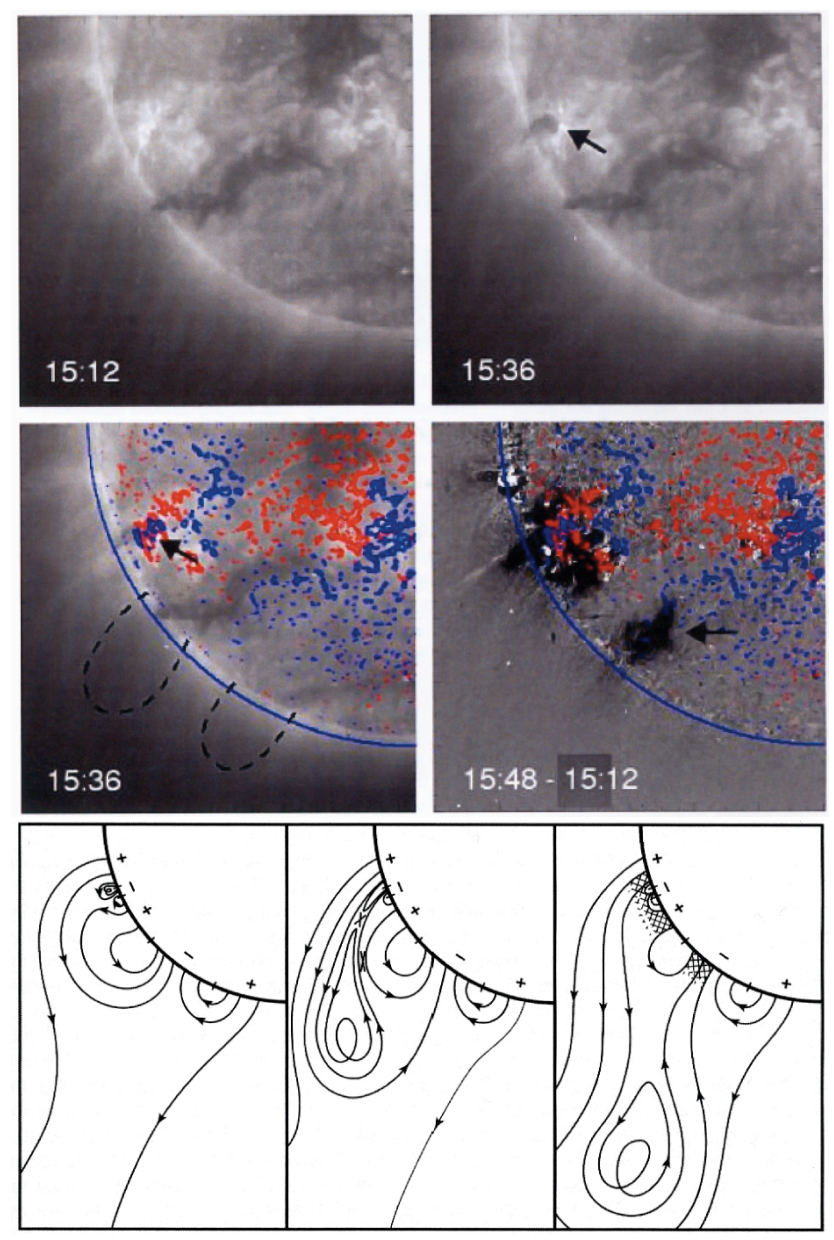

Fig. 9. From Moore and Sterling (2007) showing how a compact eruption (indicated by an arrow in the upper panel) occurring close to a streamer's base, through magnetic reconnection with an adjacent magnetic arcade, leads to a laterally more extended CME and the appearance of remote coronal dimming. In the middle panel magnetic polarities are indicated by red and blue contours, supporting the reconnection scenario (SOHO/EIT images and MDI magnetic data).

Reconnection between closed magnetic loops and field lines extended ("open") into the interplanetary space was named "interchange reconnection" by Crooker et al. (2002), who proposed this mechanism to be at work disconnecting CME field lines from the Sun. As shown by Attrill et al. (2006), Crooker and Webb (2006) and Harra et al. (2007a), disconnection indeed happens when a CME interacts with coronal hole as evidenced by uni-directional streaming of electrons measured in situ in the associated magnetic cloud.

\subsection{Reconnection with the magnetic carpet}

We have discussed the interaction of CMEs with large-scale magnetic structures such as TELs and coronal holes, but 


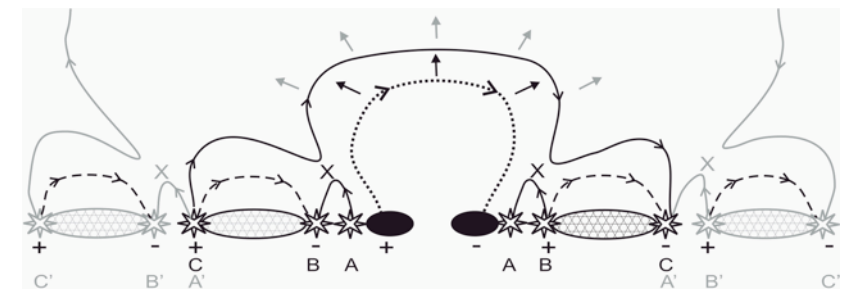

Fig. 10. Cartoon illustrating the magnetic reconnection between CME and the magnetic carpet proposed by Attrill et al. (2007a). The expanding CME (dotted line) reconnects with favourably oriented quiet-Sun magnetic loops (dashed lines), displacing the footpoints of the expanding CME (solid line). The crosses mark regions where magnetic reconnection occurs. The dotted/dashed lines show the pre-eruption magnetic structures, the black (gray) solid lines show the result of the first (subsequent) reconnections. This model provides a new explanation for the so-called "EIT-wave" phenomenon (its diffuse variety) and also explains the formation of wide-spread shallow dimming behind the wave front.

CMEs may also occur in a quiet Sun environment, like the famous CME of 12 May 1997. In such a case, many of the possibilities discussed above for enabling the CME to become large-scale do not exist! Solar magnetic fields are ubiquitous, and all over the quiet Sun the magnetic carpet consists of randomly oriented loops of different lengths and heights. In such an environment, an expanding CME must come into contact with quiet-sun loops, some of which must be expected to be favourably oriented for magnetic reconnection to occur.

A new CME evolution model discussed in Attrill et al. (2007a) considers the case where the expanding magnetic "bubble" of a CME reconnects with surrounding small-scale magnetic loops over the quiet Sun (i.e. with randomly oriented loops of different lengths and heights, as reported by Feldman et al., 1999). The reconnections lead to smallscale flare-like events and a gradual "stepping-out" of magnetic field lines originally rooted in a small source region on the Sun (Figs. 10 and 12). Such a process naturally generates a diffuse expanding bright front (known as a coronal or EIT "wave") due to the small flare-like events. Widespread coronal dimming also manifests behind the expanding diffuse bright front, since field lines rooted in the quiet Sun are successively "opened" by the reconnections to become constituents of the CME. The successive reconnections are driven by the expansion of the CME core (which is defined by the CME's over-pressure relative to its surroundings). Therefore, this model naturally and intrinsically implies that the extent of the diffuse coronal dimming matches the angular extent of the CME (Attrill et al., 2007b).

We would like to note that such a coronal EIT "wave" created by successive reconnection events does not exclude, and in fact can co-exist with an MHD shock wave formed by a fast expanding CME front (Pomoell et al., 2008), or a flare-induced blast wave (see e.g. Fig. 2 in Thompson et al., 2000b). These MHD shock waves can be identified as Moreton waves and have Type II radio burst signatures. EIT waves generated by these two different mechanisms are probably not exactly co-spatial, but since both the shock and successive reconnections are induced by the fast-expanding CME, their lower-coronal signatures should be relatively close in space. Veronig et al. (2006) has indeed observed one event (17 January 2005 CME) when the EIT wave front was about $50 \mathrm{Mm}$ ahead but roughly cospatial with the observed Moreton wave.

\section{Angular width of CMEs}

\subsection{What determines the CME's angular width?}

Recently Moore et al. (2007) argued that the final angular width of a CME can be directly related to the flaring source region. Moore et al. (2007) showed, for three very different CME events (a streamer-puff, a filament eruption and from a complex and strong magnetic field active region), that the CME's final angular width in the outer corona, $\theta_{\mathrm{CME}}$, can be reasonably well estimated from the average magnetic field strength, $B_{\text {Flare }}$, and the equivalent angular width, $\theta_{\text {Flare }}$, of the source-region flare arcade:

$B_{\text {Flare }} \approx B^{*}\left(\theta_{\mathrm{CME}} / \theta_{\text {Flare }}\right)^{2}$,

where $B^{*}$ is the field strength given by radial extrapolation of the radial field in the outer corona down to the surface of the Sun $\left(B^{*}\right.$ is estimated to be $\approx 1.4 \mathrm{G}$ ). This equation results from the pressure balance and flux conservation between the erupting CME and the surrounding open magnetic field in the outer corona. Since a low- $\beta$ plasma is assumed in the model, the dominant pressure is magnetic. Moore et al. (2007) provide a strong observationally supported argument for the "initiation site", or the region where the flare arcade is forming, being responsible for the width of the CME as opposed to the opinion of Wang et al. (2007) and Zhang et al. (2007) cited in Sect. 3.

\subsection{Synthesis - large-scale and small-scale together}

A challenging test of Attrill and co-worker's (2007a, b) CME evolution model was made by applying it to 28 October 2003 X17 flare-halo CME event (Mandrini et al., 2007). As described in Sect. 4.6, the essence of this model is that the expanding CME's field lines reconnect with field lines of surrounding magnetic structures, which may include favorably oriented loops of the magnetic carpet, quiet Sun, magnetic arcades, and active regions, depending on the surrounding magnetic environment. The reconnections form new closed loops, effectively transferring the foot-points of the outermost field lines of the CME outward, away from the original initiation site. Thus making magnetic structures over an extended area constituents of the CME. 


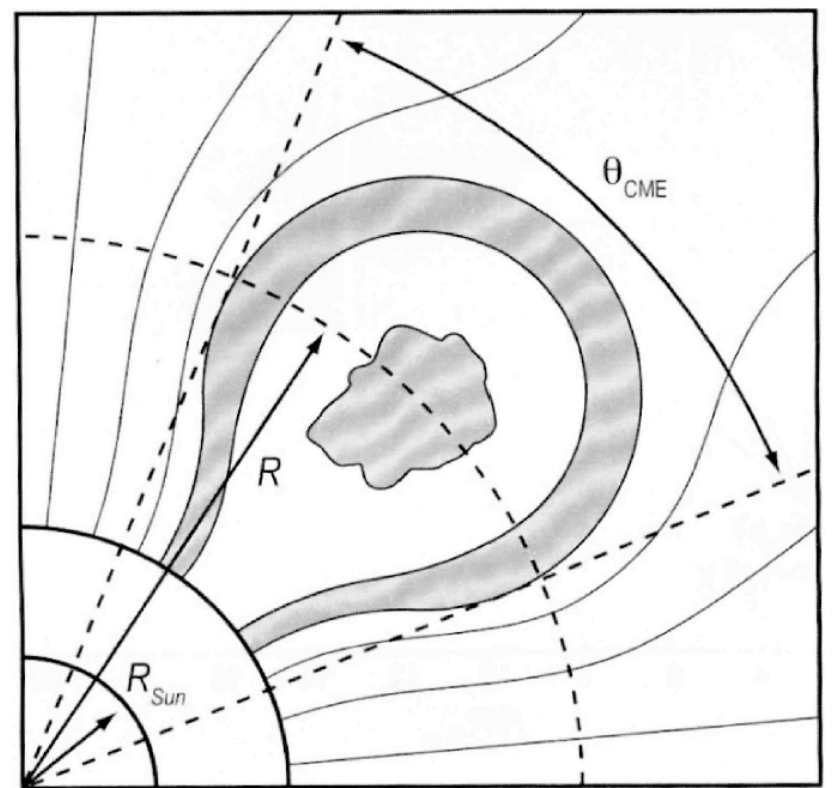

Fig. 11. From Moore et al. (2007) showing how the CMEs final angular width $\left(\theta_{\mathrm{CME}}\right)$ in the outer corona can be reasonably well estimated from the amount of magnetic flux covered by the sourceregion flare arcade due to the pressure balance of the CME with the surrounding magnetic field.

In the complex case of the 28 October 2003 CME this model allows us to understand the formation of more than 30 dimming regions created during this event (Fig. 12). There is also a coronal EIT "wave" associated with this event, most readily identified in the few patches of quiet Sun between the many different active regions and coronal holes on the solar disk. The strong lateral expansion in this case drives reconnections with the surrounding quiet Sun and larger-scale arcades, so the CME becomes large-scale, even in the low corona, as evidenced by the large spatial extent of the CMEassociated dimmings and the near-global extent of the coronal EIT "wave" bright front.

\subsection{Caveat: in general, CMEs are large-scale, but not all}

It is a paradigm in solar physics that "CMEs are large-scale events". However, it is not always the case, and in fact the majority of CMEs belong to the narrow CME category (e.g. Robbrecht, 2007) and are not observed to become large-scale in the lower corona. For example, CME source regions can be as small as an EUV bright point! Evidence for this is presented by Mandrini et al. (2005), of the smallest CME and magnetic cloud source region identified to date.

What makes the difference between a large-scale and narrow CME? It will depend to a large extent on the magnetic over-pressure which may be determined by the strength of the magnetic field region of the counterpart flare (Moore et al., 2007). Events with a high core pressure compared to
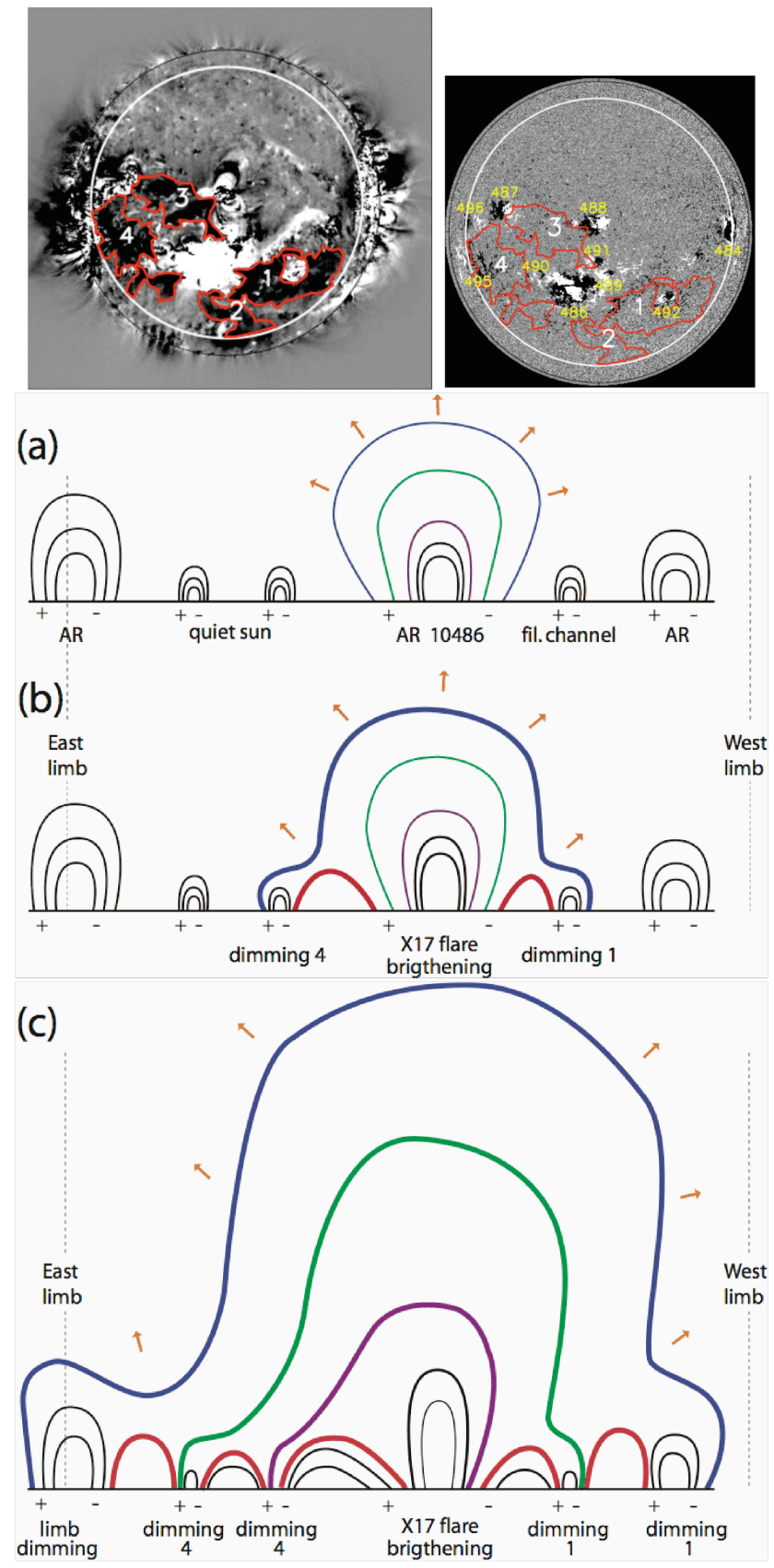

Fig. 12. A set of figures from Mandrini et al. (2007) on 28 October 2003 CME event showing how the surrounding magnetic stuctures become constituents of the large-scale CME. The upper panel shows the location of the main dimming regions (left: difference EIT; right: MDI image - the red contours enclose the main dimming regions). The lower panel shows a series of cartoons (a-c) depicting the lower-coronal evolution of this CME. The cause of formation of a large number of coronal dimming regions is explained by the progression of subsequent reconnections between the expanding CME and various magnetic structures including the magnetic carpet, ARs and a magnetic arcade straddling a filament. 
that of the surrounding magnetic environment are expected to expand more strongly in the lateral (as opposed to purely radial) direction. These CMEs are thus more likely to drive interactions with the low-coronal magnetic environment and are expected to generate lower-coronal disturbances including EIT coronal "waves" and dimming signatures that reach further away (e.g. on 28 October 2003), than events with a lower ratio of core to surrounding field pressure which will have a smaller cone-angle and will be less extended. The coronal "wave" will naturally stop when the internal pressure is no longer large enough to drive the reconnections, which effectively make the CME field lines step out of the vicinity of the initiation site.

\section{Conclusions}

We have reviewed ideas and observational results pro and contra the two conflicting views:

(i) CMEs are intrinsically non-local so that their energy supply, initiation and angular width all originate from a largescale region.

(ii) CMEs are locally initiated, and later on, during their development, they involve large-scale magnetic structures.

We found little credible observational evidence for (i) while finding much corroborating supporting evidence for (ii). Furthermore, a very recent development in CME studies, is that we now have an increasingly clear picture of how (ii) happens.

The picture we find credible is as follows: CMEs are expanding magnetic structures, whose final angular scale depends on the magnetic over-pressure in the CME (i.e. pressure difference between the CME and its surroundings), which is in turn defined by the physical conditions like magnetic shear and free energy in the CME core source region. In this sense, the final angular width of a CME is pre-defined by nature as a result of the factors discussed above. However, we conclude that CMEs become large-scale in the low corona, spanning the angular width of the CME, through interaction/reconnection with other surrounding favourably oriented magnetic structures.

This interaction (reconnection) works on different spatial scales including the

- magnetic carpet

- large-scale arcades over filament channel

- other active regions

- multi-polar systems enveloped by a streamer

- "open" magnetic field of coronal holes.

Consequence: CMEs become large-scale in the low corona through nurture. They develop to such large-scales as some of the field lines originally rooted in the flaring source region step out by successive reconnections over as wide an area as the balance between internal and external pressure allows the departing CME to expand in a lateral direction. This creates wide-spread brightenings, identified as the bright front of diffuse coronal EIT "waves", accompanied by non-thermal radio bursts, followed by dimmings. Through this process, structures over a large-scale magnetic area become CME constituents. Since magnetic reconnection between the CME and surrounding magnetic structures conserve magnetic flux, these external constituents of the CME are not expected to increase its magnetic flux (except for the overlying loops, which are forced to expand). The wide-spread coronal dimming, however, suggests that these constituents supply some mass to the CME.

Acknowledgements. We are grateful to the anonymous referees for their constructive comments, which greatly helped us to improve the paper. L. van Driel-Gesztelyi acknowledges Hungarian government grant OTKA T048961. G. D. R. Attrill is grateful to STFC for support. C. H. Mandrini and P. Démoulin acknowledge financial support from CNRS (France) and CONICET (Argentina) through their cooperative science program (No. 20326). L. K. Harra acknowledges the Leverhulme Trust for the award of a Philip Leverhulme prize. The majority of illustrations in the paper use SOHO observations. We thank the SOHO/EIT, LASCO and MDI consortia for the data. SOHO is a project of international cooperation between ESA and NASA.

Topical Editor R. Forsyth thanks two anonymous referees for their help in evaluating this paper.

\section{References}

Antiochos, S. K., DeVore, C. R., and Klimchuk, J. A.: A Model for Solar Coronal Mass Ejections, Astrophys. J., 510, 485-493, doi:10.1086/306563, 1999.

Attrill, G., Nakwacki, M. S., Harra, L. K., van Driel-Gesztelyi, L., Mandrini, C. H., Dasso, S., and Wang, J.: Using the Evolution of Coronal Dimming Regions to Probe the Global Magnetic Field Topology, Solar Phys., 238, 117-139, doi:10.1007/ s11207-006-0167-5, 2006.

Attrill, G., Harra, L. K., van Driel-Gesztelyi, L., and Démoulin, P.: Coronal "Wave": Magnetic footprint of a CME?, Astrophys. J. Lett., 656, L101-L104, 2007a.

Attrill, G. D. R., Harra, L. K., van Driel-Gesztelyi, L., Démoulin, P., and Wülser, J.-P.: Coronal "wave": A signature of the mechanism making CMEs large-scale in the low corona?, Astronomische Nachrichten, 328, 760-763, doi:10.1002/asna.200710794, 2007b.

Aulanier, G., DeLuca, E. E., Antiochos, S. K., McMullen, R. A., and Golub, L.: The Topology and Evolution of the Bastille Day Flare, Astrophys. J., 540, 1126-1142, doi:10.1086/309376, 2000.

Bemporad, A., Sterling, A. C., Moore, R. L., and Poletto, G.: A New Variety of Coronal Mass Ejection: Streamer Puffs from Compact Ejective Flares, Astrophys. J. Lett., 635, L189-L192, doi:10.1086/499625, 2005.

Bravo, S.: A Solar Scenario for the Associated Occurrence of Flares, Eruptive Prominences, Coronal Mass Ejections, Coronal 
Holes, and Interplanetary Shocks, Solar Phys., 161, 57-65, doi: 10.1007/BF00732084, 1995.

Bravo, S., Blanco-Cano, X., and López, C.: Characteristics of interplanetary magnetic clouds in relation to their solar association, J. Geophys. Res., 104, 581-592, doi:10.1029/98JA02726, 1999.

Chertok, I. M. and Grechnev, V. V.: Large-scale Dimmings Produced by Solar Coronal Mass Ejections According to SOHO/EIT Data in Four EUV Lines, Astronomy Reports, 47, 934-945, doi: 10.1134/1.1626196, 2003.

Crooker, N. U. and Webb, D. F.: Remote sensing of the solar site of interchange reconnection associated with the May 1997 magnetic cloud, J. Geophys. Res., 111, A08108, doi:10.1029/ 2006JA011649, 2006.

Crooker, N. U., Gosling, J. T., and Kahler, S. W.: Reducing heliospheric magnetic flux from coronal mass ejections without disconnection, J. Geophys. Res., 107, 1028, doi:10.1029/ 2001JA000236, 2002.

Delannée, C.: Another view of the EIT wave phenomena, Astrophys. J., 545, 512-523, 2000.

Delannée, C. and Aulanier, G.: Cme Associated with Transequatorial Loops and a Bald Patch Flare, Solar Phys., 190, 107-129, doi:10.1023/A:1005249416605, 1999.

Delannée, C., Hochedez, J.-F., and Aulanier, G.: Stationary parts of an EIT and Moreton wave: a topological model, Astron. Astrophys., 465, 603-612, doi:10.1051/0004-6361:20065845, 2007.

Feldman, U., Widing, K. G., and Warren, H. P.: Morphology of the Quiet Solar Upper Atmosphere in the $40 \mathrm{kK}-1.4$ MK Temperature Regime, Astrophys. J., 522, 1133-1147, doi:10.1086/ 307682, 1999.

Gary, G. A. and Moore, R. L.: Eruption of a Multiple-Turn Helical Magnetic Flux Tube in a Large Flare: Evidence for External and Internal Reconnection That Fits the Breakout Model of Solar Magnetic Eruptions, Astrophys. J., 611, 545-556, doi: 10.1086/422132, 2004.

Glover, A., Harra, L. K., Matthews, S. A., and Foley, C. A.: The association of transequatorial loops in the solar corona with coronal mass ejection onset, Astron. Astrophys., 400, 759-767, doi: 10.1051/0004-6361:20021886, 2003.

Hansen, R. T., Garcia, C. J., Hansen, S. F., and Yasukawa, E.: Abrupt Depletions of the Inner Corona, Publ. Astron. Soc. Pacific, 86, 500-515, 1974.

Harra, L. K. and Sterling, A. C.: Material Outflows from Coronal Intensity "Dimming Regions" during Coronal Mass Ejection Onset, Astrophys. J. Lett., 561, L215-L218, doi:10.1086/324767, 2001.

Harra, L. K., Démoulin, P., Mandrini, C. H., Matthews, S. A., van Driel-Gesztelyi, L., Culhane, J. L., and Fletcher, L.: Flows in the solar atmosphere due to the eruptions on the 15th July, 2002, Astron. Astrophys., 438, 1099-1106, doi:10.1051/0004-6361: 20052965, 2005.

Harra, L. K., Crooker, N. U., Mandrini, C. H., van DrielGesztelyi, L., Dasso, S., Wang, J., Elliott, H., Attrill, G., Jackson, B. V., and Bisi, M. M.: How Does Large Flaring Activity from the Same Active Region Produce Oppositely Directed Magnetic Clouds?, Solar Phys., 244, 95-114, doi:10.1007/ s11207-007-9002-x, 2007a.

Harra, L. K., Hara, H., Imada, S., Young, P. R., Williams, D., Sterling, A., Korendyke, C., and Attrill, G.: Coronal dimming observed with Hinode: outflows related to a coronal mass ejection,
Publ. Astron. Soc. Japan, 59, S801-S806, 2007b.

Harrison, R. A.: Solar coronal mass ejections and flares, Astron. Astrophys., 162, 283-291, 1986.

Harrison, R. A. and Lyons, M.: A spectroscopic study of coronal dimming associated with a coronal mass ejection, Astron. Astrophys., 358, 1097-1108, 2000.

Hewish, A. and Bravo, S.: The sources of large-scale heliospheric disturbances, Solar Phys., 106, 185-200, 1986.

Hudson, H. S. and Cliver, E. W.: Observing coronal mass ejections without coronagraphs, J. Geophys. Res., 106, 25 199-25214, doi:10.1029/2000JA004026, 2001.

Hudson, H. S., Acton, L. W., Alexander, D., Freeland, S. L., Lemen, J. R., and Harvey, K. L.: Yohkoh/SXT soft X-ray observations of sudden mass loss from the solar corona, in: Solar Wind Conference, pp. 88-91, 1995.

Hudson, H. S., Acton, L. W., and Freeland, S. L.: A Long-Duration Solar Flare with Mass Ejection and Global Consequences, Astrophys. J., 470, 629-635, doi:10.1086/177894, 1996.

Hudson, H. S., Lemen, J. R., and Webb, D. F.: Coronal X-Ray Dimming in: Two Limb Flares, in: Magnetic Reconnection in the Solar Atmosphere, edited by: Bentley, R. D. and Mariska, J. T., vol. 111 of Astronomical Society of the Pacific Conference Series, pp. 379-382, 1997.

Khan, J. I. and Hudson, H. S.: Homologous sudden disappearances of transequatorial interconnecting loops in the solar corona, Geophys. Res. Lett., 27, 1083-1086, 2000.

Klimchuk, J. A.: Theory of Coronal Mass Ejections, in: Space Weather, Geophysical Monograph 125, edited by: Song, P., Siscoe, G., and Singer, H., 125, 143-155, 2001.

Maia, D., Vourlidas, A., Pick, M., Howard, R., Schwenn, R., and Magalhães, A.: Radio signatures of a fast coronal mass ejection development on November 6, 1997, J. Geophys. Res., 104, 12 507-12 514, doi:10.1029/1999JA900033, 1999.

Mandrini, C. H., Pohjolainen, S., Dasso, S., Green, L. M., Démoulin, P., van Driel-Gesztelyi, L., Copperwheat, C., and Foley, C.: Interplanetary flux rope ejected from an X-ray bright point. The smallest magnetic cloud source-region ever observed, Astron. Astrophys., 434, 725-740, doi:10.1051/0004-6361: 20041079, 2005.

Mandrini, C. H., Nakwacki, M. S., Attrill, G., van Driel-Gesztelyi, L., Démoulin, P. Dasso, S., and Elliot, H.: Are CME-related dimmings always a simple signature of interplanetary magnetic cloud footpoints?, Solar Phys., 244, 25-43, 2007.

Manoharan, P. K., van Driel-Gesztelyi, L., Pick, M., and Démoulin, P.: Evidence for Large-Scale Solar Magnetic Reconnection from Radio and X-Ray Measurements, Astrophys. J. Lett., 468, L73L77, 1996.

Moore, R. and Sterling, A. C.: The Coronal-Dimming Footprint of a Streamer-Puff Coronal mass Ejection: Confirmation of the Magnetic-Arch-Blowout Scenario, Astrophys. J., 661, 543-550, 2007.

Moore, R., Sterling, A. C., and Suess, S. T.: The width of a Solar Coronal Mass Ejection and the Source of the Driving Magnetic Explosion: A Test of the Standard Scenario for CME Production, Astrophys. J., 668, 1221-1231, 2007.

Moses, D., Clette, F., Delaboudinière, J.-P., Artzner, G. E., Bougnet, M., Brunaud, J., Carabetian, C., Gabriel, A. H., Hochedez, J. F., Millier, F., Song, X. Y., Au, B., Dere, K. P., Howard, R. A., Kreplin, R., Michels, D. J., Defise, J. M., Jamar, C., Rochus, 
P., Chauvineau, J. P., Marioge, J. P., Catura, R. C., Lemen, J. R., Shing, L., Stern, R. A., Gurman, J. B., Neupert, W. M., Newmark, J., Thompson, B., Maucherat, A., Portier-Fozzani, F., Berghmans, D., Cugnon, P., van Dessel, E. L., and Gabryl, J. R.: EIT Observations of the Extreme Ultraviolet Sun, Solar Phys., 175, 571-599, doi:10.1023/A:1004902913117, 1997.

Pick, M., Maia, D., Kerdraon, A., Howard, R., Brueckner, G. E., Michels, D. J., Paswaters, S., Schwenn, R., Lamy, P., Llebaria, A., Simnett, G., Lanzerotti, L. J., and Aurass, H.: Joint Nancay Radioheliograph and LASCO Observations of Coronal Mass Ejections - II. The 9 July 1996 Event, Solar Phys., 181, 455-468, 1998.

Pick, M., Forbes, T. G., Mann, G., Cane, H. V., Chen, J., Ciaravella, A., Cremades, H., Howard, R. A., Hudson, H. S., Klassen, A., Klein, K. L., Lee, M. A., Linker, J. A., Maia, D., Mikic, Z., Raymond, J. C., Reiner, M. J., Simnett, G. M., Srivastava, N., Tripathi, D., Vainio, R., Vourlidas, A., Zhang, J., Zurbuchen, T. H., Sheeley, N. R., and Marqué, C.: Multi-Wavelength Observations of CMEs and Associated Phenomena, Space Sci. Rev., 123, 341382, doi:10.1007/s11214-006-9021-1, 2006.

Pohjolainen, S., Maia, D., Pick, M., Vilmer, N., Khan, J. I., Otruba, W., Warmuth, A., Benz, A., Alissandrakis, C., and Thompson, B. J.: On-the-Disk Development of the Halo Coronal Mass Ejection on 1998 May 2, Astrophys. J., 556, 421-431, doi: 10.1086/321577, 2001.

Pohjolainen, S., Vilmer, N., Khan, J. I., and Hillaris, A. E.: Early signatures of large-scale field line opening. Multi-wavelength analysis of features connected with a "halo" CME event, Astron. Astrophys., 434, 329-341, doi:10.1051/0004-6361:20041378, 2005.

Pomoell, J., Vainio, R., and Kissmann, R.: MHD modeling of coronal large-amplitude waves related to CME lift-off, Solar Phys., in press, 2008.

Robbrecht, E.: New techniques for the characterisation of dynamical phenomena in solar coronal images, Ph.D. thesis, Royal Observatory of Belgium, 2007.

Sterling, A. C. and Moore, R. L.: Evidence for Gradual External Reconnection before Explosive Eruption of a Solar Filament, Astrophys. J., 602, 1024-1036, doi:10.1086/379763, 2004.

Švestka, Z., Dennis, B. R., Woodgate, B. E., Pick, M., Raoult, A., Rapley, C. G., and Stewart, R. T.: Unusual coronal activity following the flare of 6 November 1980, Solar Phys., 80, 143-159, 1982.

Tang, F. and Moore, R. L.: Remote flare brightenings and type III reverse slope bursts, Solar Phys., 77, 263-276, 1982.
Thompson, B. J., Plunkett, S. P., Gurman, J. B., et al.: SOHO/EIT observations of an Earth-directed coronal mass ejection on May 12, 1997, Geophys. Res. Lett., 25, 2461-2464, 1998.

Thompson, B. J., Cliver, E. W., Nitta, N., Delannée, C., and Delaboudinière, J.-P.: Coronal Dimmings and Energetic CMEs in April-May 1998, Geophys. Res. Lett., 27, 1431-1434, doi: 10.1029/1999GL003668, 2000a.

Thompson, B. J., Reynolds, B., Aurass, H., Gopalswamy, N., Gurman, J. B., Hudson, H. S., Martin, S. F., and St. Cyr, O. C.: Observations of the 24 September 1997 Coronal Flare Waves, Solar Phys., 193, 161-180, 2000 b.

Švestka, Z., Fárník, F., Hudson, H. S., Uchida, Y., Hick, P., and Lemen, J. R.: Large-Scale Active Coronal Phenomena in YOHKOH SXT Images, I, Solar Phys., 161, 331-363, doi: 10.1007/BF00732074, 1995.

Veronig, A. M., Temmer, M., Vršnak, B., and Thalmann, J. K.: Interaction of a Moreton/EIT Wave and a Coronal Hole, Astrophys. J., 647, 1466-1471, doi:10.1086/505456, 2006.

Wang, J.-X., Zhang, Y.-Z., Zhou, G.-P., Harra, L., Williams, D., and Jiang, Y.-C.: Solar Trans-Equatorial Activity, Solar Phys., 244, 75-94, 2007.

Webb, D. F., Kahler, S. W., McIntosh, P. S., and Klimchuck, J. A.: Large-scale structures and multiple neutral lines associated with coronal mass ejections, J. Geophys. Res., 102, 24 161-24 174, doi:10.1029/97JA01867, 1997.

Wen, Y., Wang, J., Maia, D. J. F., Zhang, Y., Zhao, H., and Zhou, G.: Spatial and Temporal Scales of Coronal Magnetic Restructuring in the Development of Coronal Mass Ejections, Solar Phys., 239, 257-276, doi:10.1007/s11207-006-0181-7, 2006.

Williams, D. R., Török, T., Démoulin, P., van Driel-Gesztelyi, L., and Kliem, B.: Eruption of a Kink-unstable Filament in NOAA Active Region 10696, Astrophys. J. Lett., 628, L163-L166, doi: 10.1086/432910, 2005.

Zarro, D. M., Sterling, A. C., Thompson, B. J., Hudson, H. S., and Nitta, N.: SOHO EIT Observations of Extreme-Ultraviolet "Dimming" Associated with a Halo Coronal Mass Ejection, Astrophys. J. Lett., 520, L139-L142, doi:10.1086/312150, 1999.

Zhang, Y., Wang, J., Attrill, G. D. R., Harra, L. K., Yang, Z., and He, X.: Coronal Magnetic Connectivity and EUV Dimmings, Solar Phys., 241, 329-349, doi:10.1007/s11207-007-0229-3, 2007.

Zhou, G.-P., Wang, J.-X., Wang, Y.-M., and Zhang, Y.-Z.: Quasi-Simultaneous Flux Emergence in the Events of OctoberNovember 2003, Solar Phys., 244, 13-24, 2007.

Zhukov, A. N. and Veselovsky, I. S.: Global Coronal Mass Ejections, Astrophys. J. Lett., 664, 131-134, 2007. 